[Reseña de libros]

\title{
Nueva ecología de los medios y desarrollo ciudadano
}

Denis Renó, Luciana Renó y Carolina Campalans. (2014). Nueva ecología de los medios y desarrollo ciudadano. Bogotá: Editorial Universidad del Rosario. 181 pp. ISBN 978-958-738-529-8

El libro Nueva ecología de los medios y desarrollo ciudadano está organizado por investigadores de referencia en este ámbito de estudio. Denis Renó es un periodista, doctorado en Comunicación, post-doctorado de la Universidad Complutense de Madrid (España) y la Universidad de Aveiro (Portugal). Es profesor del curso de periodismo y del programa de postgrado en Medios y Tecnología de la Universidad Estatal Paulista (Brasil). Además, es el director académico de la Cátedra Latinoamericana Narrativas Transmedia (Rosario, Argentina) y es uno de los líderes del Grupo de Estudio sobre la nueva ecología de los medios de comunicación, un tema central de este volumen. Por su parte, Luciana Renó es una ingeniera electricista, tiene una Maestría en Procesos de Información y durante el doctorado desarrollado en la Universidad Complutense de Madrid (España) investigó en el ámbito del periodismo de datos y la narrativa Transmedia. Actualmente desarrolla el post-doctorado en Periodismo de Datos y Medios de Comunicación Sociales de la Universidad de Sao Paulo (Brasil). Carolina Campalans es periodista graduada de la Universidad de Playa Ancha (Chile) y Master en Comunicación Social por la Universidad Pompeu Fabra (España). En la actualidad es profesora y coordinadora del Programa de Periodismo y Opinión Pública de la Universidad de Rosario en Colombia.
Nueva ecología de los medios y desarrollo ciudadano presenta en cada capítulo, contenidos y puntos de vistas diversos provenientes de países como Brasil, Colombia, México, Portugal y Estados Unidos, que permiten al lector construir conocimiento y desarrollo práctico a través de ejemplos y temas relacionados con estudios sobre la nueva ecología de los medios de comunicación. Todo esto a partir de los nuevos modelos de negocio y considerando los escenarios de la comunicación social contemporánea.

En el primer capítulo, la investigadora brasileña M. Cicilia Peruzzo Krohling realiza una contextualización de la discusión sobre el desarrollo ciudadano en la configuración de una nueva ecología mediática. Para la investigadora, la cibercultura avanza señalando diversos caminos para consolidar procesos de información, producción de conocimiento y comunicación y fomentar una nueva sociedad. El capítulo “Desafíos de la comunicación popular y comunitaria en cibercultura: aproximación a la propuesta de Comunidad Emergente de Conocimiento Local" es la puerta de entrada a los debates y las reflexiones de los próximos capítulos.

Por su parte, Octavio Islas, utiliza las primeras líneas del segundo capítulo del libro para apuntar importantes ideas de Marshall McLuhan. En “McLuhan, un pensador complejo", el investigador también explica lo que es la ecología de los medios a través de autores como Neil Postman, Paul Levinson y Robert Logan. En este capítulo,
Islas genera pertinentes reflexiones sobre la velocidad en la que circula la información en múltiples pantallas que permiten la convergencia entre los viejos y los nuevos medios. En ese mismo sentido, de procesos y lenguajes emergentes en la comunicación, el siguiente capítulo titulado “Narrativa transmedia y su influencia en los procesos de comunicación", el brasileño Vicente Gosciola analiza la narrativa transmedia como una estrategia para crear productos periodísticos que llegan a audiencias conectadas que no se satisfacen con un único medio de comunicación, sino que a menudo utilizan de forma simultanea múltiples plataformas.

En el siguiente capítulo, Jorge Alberto Hidalgo Toledo complementa las reflexiones de los capítulos anteriores presentando y enumerando en “Macrotendencias de la cultura digital" las posibilidades del mundo digital, que surgen a través de los procesos de adaptabilidad de la sociedad contemporánea. Para el investigador mexicano, los nuevos medios convierten a las personas invisibles en ciudadanos visibles $y$, de esta forma, modifican la moral, los rituales y el comportamiento de los seres humanos en la sociedad.

Los portugueses Nídia Salomé Morais, San Juan Bautista y Fernando Ramos, muestran los resultados de una encuesta sobre el uso de tecnologías de la comunicación en procesos pedagógicos en el capítulo “Uso de Tecnologías de la Comunicación en contextos de aprendizaje: 
Un estudio con profesores y estudiantes en la Escuela Superior Pública portuguesa". Para los investigadores, fue posible observar la frecuencia de uso en las plataformas de gestión del aprendizaje y en las tecnologías de comunicación interpersonal, tanto por parte de los profesores como de los estudiantes.

Las discusiones sobre las nociones de hipertexto, hipermedia e interactividad están presentes en "Interfaces y narrativas de espacios e-learning: un estudio de caso del portal educativo Campus Sapo". En este artículo, Luciana Renó presenta una investigación que consolida las discusiones iniciadas en el capítulo anterior por los investigadores portugueses. En este capítulo se ofrecen fundamentos para la mejora y la continuidad en el estudio de interfaces interactivas en el campo de la educación para el desarrollo social.

Robert K. Blechman, profesor y media-ecologista, presenta en el capítulo titulado " $¿ E l$ tedio es el mensaje? La comunicación y la creación con Nuevas Redes Sociales" la experimentación en la producción de libros de ficción, abordando las teorías de comunicación que son producidas interactivamente a través del uso de Twitter. De esta forma, el investigador se suma a la investigación sobre nuevos ecosistemas mediáticos, la creatividad y la literatura.

A continuación, el investigador Denis Renó muestra los resultados de su investigación experimental con documentales transmedia. En el capítulo "Participación ciudadana en el documental transmedia gallego-portugués", el autor presenta algunas teorías acerca de la nueva ecología de los medios de comunicación y muestra conceptos sobre un modelo de documental poco explorado por los investigadores del campo audiovisual.

“La televisión digital pública en Brasil a favor del tele-periodismo ciudadano" es el siguiente capítulo del libro. La discusión propuesta por los brasileños Francisco Machado Filho y Mayra Fernanda Ferreira proporciona información sobre el desarrollo de un formato

\section{NUEVA ECOLOGÍA DE LOS MEDIOS Y DESARROLLO CIUDADANO}
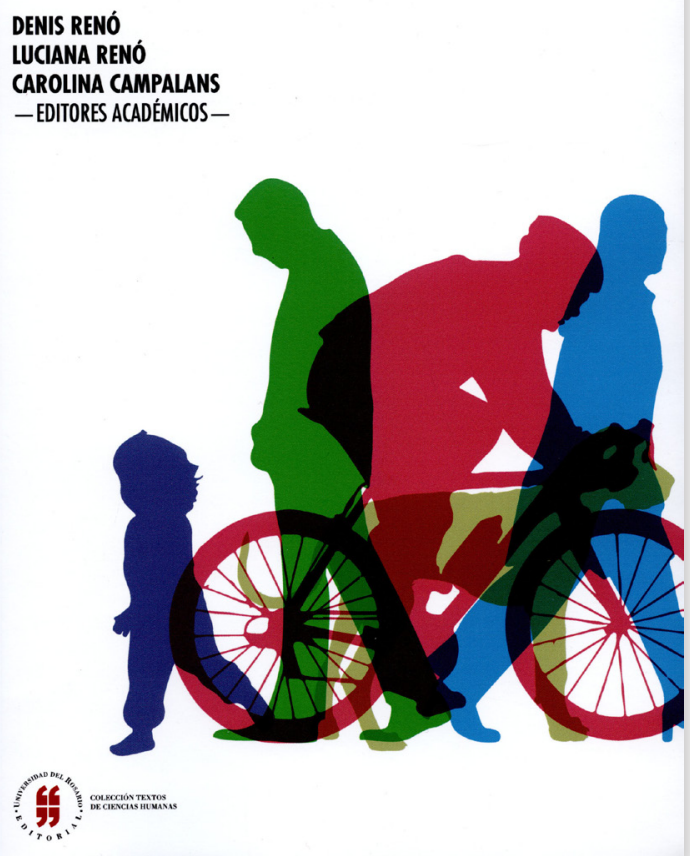

recursos educativos abiertos. Bajo el título "Superman y su potencial para los recursos educativos abiertos (REA)", el capítulo tiene como base fundamental las teorías de las nuevas narrativas comunicacionales y muestra claramente al lector que las personas pueden aprender en su tiempo a través de los vídeos disponibles en YouTube.

Por medio de múltiples lugares los investigadores provenientes de diversos países, teorías y prácticas presentan al lector de Nueva ecología de los medios y desarrollo ciudadano las identidades, los desafíos y oportunidades que dan forma al nuevo ecosistema de medios de comunicación. En el capítulo final, "Ciudadanía, democracia y periodismo sobre tecnología: un debate sobre la reforma del Link Estado", la investigadora brasileña Andressa Kikuti se refiere a la discusión sobre el papel del periodismo en la ciudadanía, y en el desarrollo y conocimiento de la nueva ecología de los medios a partir de las tecnologías emergentes. televisivo que promueve la ciudadanía a través de la televisión digital interactiva. Para ello, los investigadores hacen un estudio sobre la televisión digital en Brasil y sus innumerables posibilidades. En el capítulo siguiente, los investigadores brasileños Andrea Cristina Versuti y David Daniel Alves da Silva presentan un estudio transmediático sobre cómo la franquicia Superman apoya la educación y la ciudadanía a través de

\section{Danilo Leme Bressan}

Doctorando en Mídia e Tecnologia Universidade Estadual Paulista, Brasil. danilo.bressanagmail.com

\section{Cómo citar:}

Leme, D. (2016). [Reseña de libro] Nueva ecología de los medios y desarrollo ciudadano. Comunicación y Medios, 25 (34), 116 - 117.

DOI: 10.5354/0719-1529.2016.42556 